\title{
Aprovechamiento del estiércol caprino como recurso biomásico para la producción de biogás tomando como referencia a la comunidad Yutaho ubicado en Cuatro Vías, La Guajira: Revisión
}

Yurleys Paola Barros Gómez', Yeraldine Elvira Dangond Rodríguez², Marlon Bastidas Barranco³

\section{Resumen}

El uso de combustibles fósiles y la tala indiscriminada de la leña a través de los años se ha convertido en un fuerte problema de contaminación que ha traído como consecuencia el cambio climático y, por ende, el calentamiento global; por esta razón, los gobiernos buscan sustituir estos combustibles por fuentes de energías renovables que brinden una sostenibilidad. En este documento de revisión se hizo un enfoque sobre la energía obtenida a partir de la biomasa, como el excremento de especies caprinas, vacunas, gallinazas y porcinas. En este caso de estudio se consideró el estiércol caprino, cuyos elementos de evaluación se proyectaron a la comunidad Yutaho, ubicada en Cuatro Vía, La Guajira. Esta comunidad cuenta con 95 cabezas de especies de cabras, que producen $152 \mathrm{~kg} /$ día. Se concluyó que, de acuerdo con lo investigado, esta alternativa renovable, como lo es la biomasa sólida residual animal, puede aprovecharse de forma eficiente para la producción de biogás por medio de biodigestores en comunidades pequeñas.

Palabras clave: biodigestores, biogás, biomasa, combustibles fósiles, energías renovables. 


\section{Introducción}

En los últimos años se ha observado un crecimiento energético en algunos países del mundo, cuyo aumento es, desde los 2000 millones de toneladas equivalentes de petróleo (Mtep) hasta los 12500 Mtep, de 1960 hasta la actualidad (Villanueva, 2012). Este crecimiento energético está directamente relacionado con la alta tasa de urbanización que, por su parte, viene acompañado de la industrialización, el crecimiento económico y el desarrollo, por lo tanto, provoca un aumento acelerado en el consumo de energía (Bakirtas y Gokce, 2018).

La mayoría de los países en el mundo dependen de combustibles fósiles no renovables como su fuente principal de energía (Badii, Guillen y Abreu, 2016); algunos de los países que consumen mayor energía en el planeta son China, EE. UU., Rusia, India, Japón, Canadá, Alemania, Brasil, Francia y Corea del Sur, constituyendo un $64,6 \%$ de la energía primaria consumida a nivel mundial (Shahbaz et al., 2018). Otro factor menos importante relacionado con esta problemática es el aumento de la población que provoca un consumo excesivo de energía, debido a la necesidad que tienen las ciudades de generar grandes cantidades de esta para realizar cada una de sus actividades.

Históricamente, los países desarrollados son los que han producido la mayoría de los gases de efecto invernadero (GEI), mientras que en los últimos años la proporción de las emisiones de estos gases han sido superadas por los países en desarrollo, aumentando muy rápidamente (Shahsavari y Akbari, 2018). Los gases de efecto de invernadero son los responsables del cambio climático que a su vez han traído como consecuencia el calentamiento global que enfrenta el planeta en la actualidad (Quintero y Quintero, 2015).

Por todo lo anterior, es necesario el estudio del potencial energético de los recursos renovables a partir del enfoque de su aprovechamiento en la producción de combustibles, siendo una excelente alternativa para minimizar los problemas ambientales que amenazan al mundo, como el cambio climático, la contaminación atmosférica en diversas ciudades, la contaminación de agua, los residuos radiactivos y la lluvia ácida (Solano, 2015). La mayoría de las tecnologías en energía limpia han sido implementadas en países altamente industrializadas, especialmente los 
Estados Unidos, Japón y la Unión Europea (Miller y Visicdi, 2016).

América Latina cuenta con un gran potencial de energías renovables que, según estimaciones recientes, revelan que la región puede producir más de 78 petravatios-hora $(\mathrm{PWh})$ a partir de energía solar, eólica, marina, geotérmica y biomásica (Vergara et al., 2014). Brasil es uno de los países más destacados en Latinoamérica en cuanto al uso de energías renovables que, según datos de la Empresa de Pesquisa Energética (EPE), en este país el $85,4 \%$ de la energía que se consume deriva de fuentes renovables, sin embargo, la mayor parte de esta energía es hidroeléctrica y en poca proporción es utilizada la biomasa (Pérez, 2017), siendo esta última, según algunas organizaciones y entidades en el mundo, muy importante para la producción sostenible de energía en el futuro.

"Colombia es reconocida a nivel mundial como uno de los países más ricos en cuanto a recursos naturales" (Bolívar y Hernández, 2013), por tal razón se convierte en una excelente alternativa para hacer aprovechamiento de sus recursos renovables y, espacialmente, de su biomasa.
La biomasa, en términos energéticos, son aquellos recursos biológicos de origen vegetal, animal o producto de su transformación, de los cuales se puedan obtener combustibles energéticos, también denominados biocombustibles (Arévalo, 2015). Se conoce como la forma más antigua de energía aprovechada por los seres humanos, esencialmente las ramas y troncos de los árboles que producían calor y luz al momento de ser utilizados por medio de combustión directa (Oviedo et al., 2015).

Existen varios tipos de biomasa como la natural, residual, excedentes de cosechas y, por último, los cultivos energéticos (Fernández et al., 2015). Para este caso el tipo de biomasa que se analiza es la biomasa residual,

teniendo en cuenta que en el país existen estudios preliminares sobre esta biomasa, dentro de las cuales se destaca para su aprovechamiento solamente el bagazo de caña, la cascarilla de arroz, el cuesco, y la fibra de palma de aceite, residuos de la industria maderera y residuos de cosechas. (Pérez y Salazar, 2015)

Por lo tanto, los residuos producidos por los animales, como el estiércol, son 
muy poco usados para la generación de energía y, según sus características, se convierte en una excelente materia prima para la digestión anaeróbica debido a su alto contenido total de nitrógeno y la estabilidad de la fermentación (Zhang et al., 2013).

En Colombia, el departamento de La Guajira cuenta con la mayor cantidad de ganado caprino-ovino en el país (Quintero et al., 2010), con una población de $80,8 \%$ de caprinos (Cardona, 2017) todo esto es debido a sus características topográficas, climáticas, a la adaptabilidad de esta especie en zonas áridas y a la presencia de comunidades wayuu en las zonas que se dedican a la cría de estos animales. La generación energética en La Guajira empezó a tomar fuerza en los últimos años, un ejemplo claro de esto es el Parque Eólico Jepírachi, que fue ejecutado por Empresas Públicas de Medellín (EPM), convirtiéndose en el primer proyecto que Colombia registró oficialmente ante las Naciones Unidas para su estrategia de cambio climático (EPM, 2018). A pesar de que la huella energética de Colombia es doce veces menor que la de Estados Unidos, cinco veces menor que la de la Unión Europea y tres veces menor que la de China, ha experimentado en los últimos años un crecimiento energético como consecuencia de su continuado avance económico (Cadavid y Bolaños, 2015), lo que implica el uso de su potencial energético para suplantar los combustibles fósiles.

Teniendo en cuenta lo anterior, Colombia goza de suficiente biomasa como materia prima para su aprovechamiento en la generación de energía limpia. En cuanto al departamento de La Guajira, este posee un gran potencial energético, tomando ventaja de su liderazgo en la población de caprinos, haciendo uso de los subproductos para la generación de biogás, convirtiéndose en una excelente alternativa para su aplicación en zonas rurales en las que los servicios públicos son inexistentes y siendo una seria posibilidad para suplir algunas necesidades en comunidades de difícil acceso a las energías convencionales (Ordóñez, 2010).

Por su parte, el biogás es un recurso de energía renovable que se produce a partir de la descomposición de residuos orgánicos en condiciones anaerobias, está compuesto principalmente por metano $(60 \%)$ y dióxido de carbono (35-40\%) (Zareei, 2018).

La digestión anaerobia es el conjunto de procesos bioquímicos 
desarrollados en un ecosistema libre de oxígeno que logra la transformación y estabilización de la materia orgánica biodegradable realizada por varios grupos de microorganismos donde el producto final es el Biogás. (Cendales, 2011)

Este documento trata básicamente de una revisión teórica para el aprovechamiento del estiércol caprino como recurso biomásico para la producción de biogás, tomando como referencia a la comunidad Yutaho ubicada en Cuatro Vías, La Guajira, en la cual se realizan comparaciones de la biomasa sólida a nivel mundial, así como también se analizan los diferentes tipos de biodigestores que se utilizan para la generación de biogás mediante el aprovechamiento del estiércol, teniendo en cuenta el potencial de biomasa producida en el departamento de La Guajira. Por último, se escoge una de las alternativas más eficientes para la obtención de biogás, con el fin de garantizar la generación de este para ser utilizado por las comunidades rurales, reemplazando la leña que es talada para satisfacer algunas necesidades por falta de prestación de servicios públicos $\mathrm{y}$, de este modo, contribuyendo a la conservación del medio ambiente.
Otro aspecto importante es la falta de información y estudios relacionados sobre el aprovechamiento del estiércol caprino como una fuente para la obtención de biogás, considerando que el departamento de La Guajira cuenta con la suficiente biomasa solida animal para ser transformada y de esta forma darle un uso adecuado.

\section{Metodología}

El caso presentado se desarrolló en el área proyecto comunidad Yutaho, ubicada en Cuatro Vías, con coordenadas $11^{\circ} 24>43.4 » \mathrm{~N} \quad 72^{\circ} 24>33.7 » \mathrm{~W} \quad$ en jurisdicción del municipio de Maicao, La Guajira, Colombia, como se muestra en la figura 1.

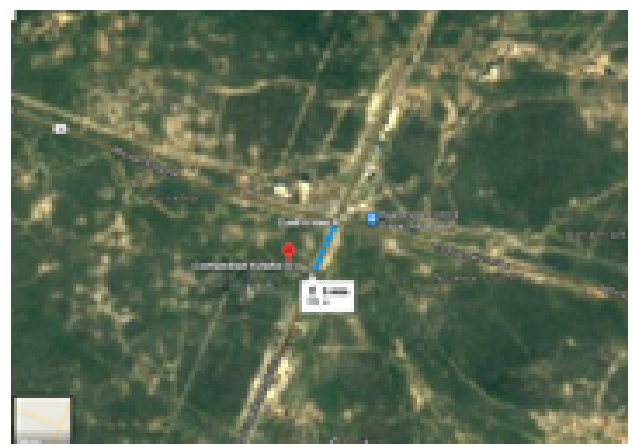

Figura 1. Área de estudio; delimitación geográfica de la comunidad Yutaho Fuente: Google Maps, 2018. 
La revisión teórica para el aprovechamiento del estiércol caprino como recurso biomásico para la producción de biogás se realizó de tipo descriptivo, con ella se investigaron varias fuentes documentales, teniendo en cuenta condiciones similares a la de una comunidad. Para el cumplimiento de los objetivos de este documento se desarrollaron las siguientes fases:

\section{Análisis de la información relacionada con los recursos biomásicos proveniente de los caprinos para la producción de biogás a nivel mundial}

El análisis sobre los recursos biomásicos se realizó con el propósito de tener en cuenta la importancia que tiene la biomasa a nivel mundial y qué tan eficiente es el momento de producir biogás; esta se elaboró de forma descriptiva por medio de la literatura, en la cual se realizaron cuadros comparativos según la interpretación de cada autor, en los que se tiene en cuenta el número de animales (caprinas, vacunas, porcinas y gallinazas) presentes en comunidades rurales y sobre la cantidad de excremento que generan estas especies en $\mathrm{kg} /$ día, respectivamente; se desarrolló un análisis por medio de la interpretación de los datos obtenidos.

Investigación de diseños de biodigestores para la estimación de la eficiencia energética de la biomasa generada por los caprinos

La investigación de diseño de biodigestores se realizó con la intención de tener en cuenta los tipos de biodigestores que existen para la producción de biogás, también para conocer cuál de estos es el más adecuado a la hora de implementarlo en comunidades rurales, teniendo en cuenta su rentabilidad y eficiencia a nivel mundial; lo anterior se ejecutó de forma descriptiva por medio de la literatura que soporta a dicha investigación.

\section{Definición de estrategia teórica para el reemplazo de la leña por biogás derivados del estiércol caprino en comunidades con condiciones similares a la de Yutaho, La Guajira}

Se realizó con el propósito de mejorar la calidad de vida de comunidades rurales y del medio ambiente, puesto que algunas comunidades utilizan la leña para realizar sus actividades de cocción, 
pero esta trae como consecuencias problemas ambientales tale como desforestación, polución de partículas de cenizas y descomposición en el suelo, entre otras.

Las estrategias se realizaron de forma descriptiva por medio de la literatura, se realizaron cuadros comparativos y figuras, en las que se logró tener en cuenta el poder calorífico de los precursores de combustible y de la energía producida por estos, las comparaciones se hicieron con el fin de establecer el remplazo de los precursores de combustible y de este modo evitar la desforestación.

En cuanto al modelo matemático para las respectivas comparaciones, inicialmente se estiman las cantidades de masa de estiércol por cabeza según el tipo de especie, para ello se emplea la ecuación 1.

$$
m_{i}=\frac{\dot{m}_{i}}{\dot{N}_{i}}
$$

En la que $m_{i}$ son los kg de estiércol por cada cabeza de la especie $i, \dot{m}_{i}$ son los kg de estiércol por día de la especie $i$ y $\hat{N}_{i}$ es el numero de cabezas registradas por día de la especie $i$.

Para el cálculo del poder calorífico según el tipo de especie se utiliza la ecuación 2.
$P C_{i}$

$=V_{b i} *$ prom $_{p c b}$

$P C_{i}$ es el poder calorífico por cada especie $i, V_{b i}$ es el volumen de biogás que requieren cada especie $i$ en $\left(\mathrm{m}^{3} / \mathrm{kg}\right)$, y prom $_{p c b}$ es el promedio del poder calorífico de biogás en $\mathrm{MJ} / \mathrm{m}^{3}$.

El valor del poder calorífico del biogás es 19.7-23 MJ de energía calórica por $\mathrm{m}^{3}$ de gas (Cunalata, 2017).

Para el cálculo de la energía producida por las especies se usa la ecuación 3.

$$
E_{i}=m_{i} * P C_{i}
$$

$E_{i}$ es la energía producida por cada especie $i, m_{i}$ son los kilogramos de estiércol por cada cabeza de la especie $i$ y $P C_{i}$ es el poder calorífico por cada especie $i$.

Por último, se utiliza la ecuación 4, para hallar la energía de los precursores de los combustibles.

$$
\begin{aligned}
& E_{p e b} \\
& =m_{p c b} * P C_{p c b}
\end{aligned}
$$

$E_{p c b}$ es la energía producida por los precursores de los combustibles, $m_{p c b}$ son los $\mathrm{kg} /$ hectáreas de los precursores y $P C_{p c b}$ es el poder calorífico de los precursores de los combustibles en $\mathrm{MJ} / \mathrm{kg}$. 


\section{Resultados}

Para el cumplimiento de los resultados de este documento se desarrollaron tres fases que se describen a continuación:

\section{Análisis de la información relacionada con los recursos biomásicos provenientes de los caprinos para la producción de biogás a nivel mundial}

En la tabla 1 se observa el promedio de cabezas para diferentes tipos de animales que permiten la comparación de los aportes energéticos provenientes del estiércol que producen cada una de ellas, tomando como referencia a la comunidad Yutaho. Se observa que la especie con mayor número de cabezas es la gallinaza, con un promedio 16484 para la generación de biogás, esto puede corresponder al poco excremento que disponen, teniendo en cuenta que este tipo de aves depositan $0.18 \mathrm{~kg} /$ día de excremento (Inca, 2016), seguida del vacuno con 174 cabezas y las especies caninas con 120 cabezas y, por último, las especies porcinas con 84 caprinas y 72 cabezas.

Tabla 1. Cantidad de cabezas de diferentes tipos de animales utilizados en diferentes trabajos para producción de biogás

\begin{tabular}{|l|c|c|c|c|c|}
\hline Especies & $\begin{array}{c}\text { N. }{ }^{\circ} \text { cabezas } \\
{[\text { Ref.] }}\end{array}$ & $\begin{array}{c}\text { N. }{ }^{\circ} \text { cabezas } \\
{[\text { Ref.] }}\end{array}$ & $\begin{array}{c}\text { N. }{ }^{\circ} \text { cabezas } \\
{[\text { Ref.] }}\end{array}$ & $\begin{array}{c}\text { N. }{ }^{\circ} \text { cabezas/ } \\
\text { comunidad } \\
\text { Yutaho }\end{array}$ & $\begin{array}{c}\text { Promedio de N. } \\
\text { de cabezas/co- } \\
\text { munidad Yutaho }\end{array}$ \\
\hline Caprinas & $80[26]$ & $40[27]$ & - & 95 & 72 \\
\hline Vacunos & $10[28]$ & $450[29]$ & $63[26]$ & - & 174 \\
\hline Porcinos & $86[30]$ & $163[31]$ & $2[32]$ & - & 84 \\
\hline Gallinazas & $4800[26]$ & $28167[33]$ & - & - & 16484 \\
\hline Caninas & $120[34]$ & - & - & - & 120 \\
\hline
\end{tabular}

\section{Fuente: elaboración propia.}

En el caso de las especies caninas se puede argumentar que, al realizar la investigación, los procesos biomásico con este tipo de especies no son comunes de implementar. Con respecto a las especies caprinas, se encontraron dos estudios, como se puede observar en la tabla 1 , esto significa que se pueden producir biogás a partir del diseño de biodigesdores en la comunidad Yutaho con las 95 cabezas caprinas disponibles. 
En la tabla 2 se observa el promedio de la cantidad de estiércol en $\mathrm{kg} /$ día que disponen las especies investigadas para producir biogás a través de un biodigestor. Se evidencia que la especie que más genera excremento son los vacunos porque, de acuerdo con la tabla 1 y 2, se encontró que por cada diez vacunos se produce $60 \mathrm{~kg} /$ día. En total, una especie genera $6 \mathrm{~kg} /$ día, esto puede corresponder a su peso promedio que equivale $300 \mathrm{~kg}$ (ver tabla 3); por consiguiente, los vacunos son la especie que más se utiliza para la producción de biogás, esto se debe a su producción de biomasa que, de acuerdo con lo investigado, cuenta con una cantidad promedio de $3278 \mathrm{~kg} /$ día por cada 174 cabezas promedio.

Tabla 2. Promedio de la cantidad de biomasa en ( $\mathrm{kg} / \mathrm{día}$ ) que disponen las especies en las comunidades rurales investigadas y en la comunidad Yutaho

\begin{tabular}{|l|c|c|c|c|c|}
\hline Especies & $\begin{array}{c}\text { Cantidad de } \\
\text { estiércol diario } \\
{[\text { Ref.] }} \\
(\mathrm{kg} / \text { día })\end{array}$ & $\begin{array}{c}\text { Cantidad } \\
\text { de estiércol } \\
\text { diario [Ref.] } \\
(\mathrm{kg} / \text { día })\end{array}$ & $\begin{array}{c}\text { Cantidad de } \\
\text { estiércol } \\
\text { diario } \\
{[\mathrm{Ref}](\mathrm{kg} /} \\
\text { día })\end{array}$ & $\begin{array}{c}\text { Cantidad } \\
\text { de estiércol } \\
\text { diario/ } \\
\text { Comunidad } \\
\text { Yutaho }(\mathrm{kg} / \\
\text { día })\end{array}$ & $\begin{array}{c}\text { Promedio de } \\
\text { la cantidad de } \\
\text { estiércol } \\
\text { diario/ } \\
\text { comunidad } \\
\text { Yutaho }(\mathrm{kg} / \\
\text { día) }\end{array}$ \\
\hline Caprinas & $128[26]$ & $27[27]$ & - & $152[26]$ & 102,3 \\
\hline Vacuno & $60[28]$ & $6750[29]$ & $3024[26]$ & - & 3278 \\
\hline Porcinos & $202,1[30]$ & $175,1[31]$ & $4[32]$ & - & 127,07 \\
\hline Gallinazas & $0,48[26]$ & $2732,20[33]$ & - & - & 1366,34 \\
\hline Canina & $20[34]$ & - & - & - & 20 \\
\hline
\end{tabular}

Fuente: elaboración propia.

Luego le siguen las gallinazas con un valor de $1366,34 \mathrm{~kg} / \mathrm{dí} a$, esto corresponde, de acuerdo con lo investigado, en la tabla 1 utilizan 28167 y 4800 cabezas, esta cantidad pertenece a la de una provincia (Altamirano, 2017) y a la de una avícula de gallinas ponedoras de huevos, por este motivo, son más elevados en comparación con las demás especies, las cuales son tomadas en comunidades rurales pequeñas.

En el caso de las especies caprinas, se cuentan con 40 cabezas de cabras que generan $27 \mathrm{~kg} /$ día, de acuerdo al informe 
investigado, de los $27 \mathrm{~kg}$ /día disponen de $9 \mathrm{~kg}$ para alimentar el biodigestor, en el que la producción fue de 2,4 $\mathrm{m} 3$ de gas diario y obtuvieron $1,68 \mathrm{~m} 3$ de metano, esto soporta el abastecimiento medio mensual de una familia (Follari y Torres, 2014). En el caso de la comunidad Yutaho, esta posee 95 cabezas de cabras que producen $152 \mathrm{~kg}$ /día; lo que indica que, a mayor número de cabezas, la cantidad de estiércol es más elevada; por consiguiente, la generación del biogás puede ser mayor (Navarro, 2015).

\section{Investigación de diseños de biodigestores para la estimación de la eficiencia energética de la biomasa generada por los caprinos}

Al hacer énfasis en un contexto general encontramos una revisión que tiene como propósito la aplicación de los biodigestores para producir biogás en las viviendas de zonas rurales de América Latina, mostrando este método como una tecnología limpia y considerada con el medio ambiente, así como para poder ayudar a las comunidades rurales, mejorando sus condiciones de vida y satisfaciendo sus necesidades básicas (Garfí et al., 2016), por otro lado, se encontró un estudio, en el cual muestra una metodología para proporcionar el análisis $\mathrm{y}$ cálculo de las medidas para la construcción de biodigestores de cúpula fija, estos diseños permiten eliminar los malos olores, reducir la carga contaminante, mejorar la capacidad fertilizante del material, y, por último, se genera una energía renovable como el biogás. (Campos, 2011)

De acuerdo con lo anterior, se encontró en la Universidad Francisco de Paula Santander, Ocaña, la construcción y diseño de un biodigestor anaeróbico, este con el propósito de aprovechar la biomasa energética, donde utilizan el abono orgánico, biogás y biol consecuentes del proceso digestivo, el cual se ejecutó por medio de bacterias sin presencia de oxígeno molecular dentro del reactor (Navarro, 2015). Desde otro punto de vista se encuentra la ejecución de un biodigestor continuo en la finca la Poderosa para la obtención de biogás a partir de estiércol, en donde la elaboración del biodigestor se manejó en un tanque de polietileno de 500 litros y se consideraron diferentes componentes (Chillo y Paguay, 2015). Así mismo, el siguiente artículo presenta la implementación y el diseño de un biodigestor continuo de un prototipo de 
bolsa para la realización de biogás a partir de heces canicas; este fue diseñado en la localidad de Tunja, municipio de Oicatá. Para la construcción se concretaron las principales características físicoquímicas de las heces caninas y las situaciones ambientales del municipio (Rodríguez y García, 2017).

Otro diseño es el desarrollo de un biodigestor tubular para obtener biogás en la hacienda Santa Mónica ubicada en la comunidad Atapo, Santa Cruz, cantón Guamote, provincia de Chimborazo; este se realizó a partir de residuos orgánicos de ganado vacuno generados en la comunidad con el propósito de reducir la contaminación producida por los desechos (Calderón, 2015). De igual modo, se encontró el modelo de diseño del sistema de biogás óptima (OBSDM). Este modelo esta propuesto para ser utilizado como un instrumento para la toma de decisiones en cuanto al crecimiento del potencial para técnicas de biogás en diferentes aplicaciones en África subsahariana (SSA). La herramienta de toma de decisiones evidencia el diseño más adecuado de biodigestor fijo basado en los insumos (Smitha, Schroenn y Blignaut, 2014). Otro modelo a seguir es el establecido en la finca El Recodo, el cual está encaminado a formar mejoras al método convencional de digestión anaerobio, usando los residuos generados por los bovinos para producir biogás (Bernal y Quintero, 2016).

Por otro lado, se ejecutó el diseño de un biodigestor tubular de flujo continuo de polietileno (anaeróbico) por ser el más apropiado a las necesidades del Rancho Guadalupe, para la obtención de biogás, a partir de excretas de ganado vacuno y, además, se elaboró un diseño de invernadero que cubrirá al biodigestor para incrementar la temperatura. (Toscano, 2016)

También se hace referencia al diseño de un biodigestor casero, utilizando estiércol bovino, para aprovecharlo como energía renovable, transportando su conversión de excremento a un producto de consumo masivo, como lo son el biocombustible y el biogás, por medio del uso de tecnologías y equipos adecuados (Cruz, 2014).

En cuanto a la siguiente investigación se implementó un biodigestor para adquirir biogás mediante de residuos orgánicos de especies vacunos producidos en el Criadero Jersey Chugllin situado en el cantón Chambo, región de Chimborazo, por el cual se busca 
reducir la contaminación formada por los desechos. (Lara, 2016)

De igual forma, se investigó un proyecto práctico en el cual hubo intención de valorar la producción de biogás a partir del diseño de un biodigestor cilíndrico que produce por períodos 233 litros de volumen de estiércol porcino y bovino. También se experimentaron diferentes variables como tiempo de retención, temperatura ambiente, presión, cantidad de biogás generado y pH (Durazno, 2018). Para el diseño del biodigestor tubular, este fue de siete metros de longitud por un metro de diámetro, con una obtención de biogás de 1,37 m3. Se realizó, además, un análisis costo beneficio, el cual fue viable para la ejecución del biodigestor en el rancho (Bautista, 2016). Con respecto a los artículos anteriormente mencionados, se puede resaltar que los biodigestores son ampliamente utilizados para la generación de biogás por medio del estiércol, especialmente en países de Latinoamérica como Ecuador, Colombia y en Centroamérica como México, los cuales cuentan con pequeñas comunidades rurales, en las que indican que el diseño de biodigestor tubular es el más adecuado para implementar en estas zonas rurales. Para el sustento del biodigestor, de acuerdo con lo investigado, se deben tener en cuenta algunos factores como lo son la temperatura, el tiempo de retención y la relación del sustrato (excremento) y el agua requerida, esto ayuda a determinar la cantidad de biogás producido y la capacidad del biodigestor (Yauyo, 2016).

\section{Definición de una estrategia teórica para el reemplazo de la leña por biogás derivados del estiércol caprino en comunidades con condiciones similares a la de Yutaho en La Guajira}

En la tabla 3 se observa la cantidad de estiércol producida por cada especie y el peso promedio de estos, la energía generada por estas y, por último, el poder calorífico en relación con el volumen del biogás. De acuerdo con los valores obtenidos, la cifra que, evidentemente, sobresale respecto a los demás valores es de 5,14 MJ/cabezas, que corresponde a los vacunos según su energía producida; en segundo lugar, encontramos la especie porcina con una energía producida de $3.02 \mathrm{MJ} /$ cabezas, esto se debe a la cantidad de excremento que estas generan. 
Tabla 3. Energía producida por cada especie expresada en MJ/cabezas

\begin{tabular}{|c|c|c|c|c|c|}
\hline Especies & $\begin{array}{c}\text { Peso promedio } \\
\text { de las especies } \\
(\mathrm{kg})\end{array}$ & $\begin{array}{c}\text { Cantidad de } \\
\text { estiércol kg/ } \\
\text { cabeza }\end{array}$ & $\begin{array}{c}\text { Volumen de } \\
\text { biogás hú- } \\
\text { medo } \mathrm{m}^{3} / \mathrm{kg}\end{array}$ & $\begin{array}{c}\text { Poder calo- } \\
\text { rífico MJ/kg }\end{array}$ & $\begin{array}{c}\text { Energía } \\
\text { producida } \\
\text { MJ/cabezas }\end{array}$ \\
\hline Caprino & $40[26]$ & 1,6 & $0,05[45]$ & 1,07 & 1,7 \\
\hline Aves & $1,5[26]$ & $1 \times 10^{-4}$ & $0,08[45]$ & 1,712 & $1,7 \times 10^{-4}$ \\
\hline Vacuno & $300[28]$ & 6,0 & $0,04[45]$ & 0,856 & 5,14 \\
\hline Porcino & $50[32]$ & 2,35 & $0,06[45]$ & 1,284 & 3,02 \\
\hline
\end{tabular}

Fuente: elaboración propia.

Los caprinos poseen una energía producida de 1,7 MJ/cabezas, que puede corresponder a la cantidad de excremento, que es de $1.6 \mathrm{~kg} /$ cabezas; también puede deberse al volumen del biogás en $\mathrm{m} 3 / \mathrm{kg}$, que corresponde a 0,05 , pero su poder calórico, de acuerdo con lo establecido en la tabla 3, es de $1,07 \mathrm{MJ} / \mathrm{kg}$, esto significa que es rentable producir biogás con este tipo de excrementos caprinos y al utilizar un mayor número de cabezas se generaría una cantidad alta de excrementos, por lo tanto, la energía obtenida sería más elevada. Por último, las aves presentan un 1,7x10-4 de energía producida, a pesar de contar con el valor más bajo, son la que mayor poder calorífico poseen con un total de 1,712 , esto puede estar relacionado con su volumen húmedo que tiene un valor de $0,08 \mathrm{~m} 3 / \mathrm{kg}$, siendo este el mayor en comparación con los demás.

En la tabla 4 se observa el poder calorífico (superior, inferior y medio) y la energía producida de los precursores de combustibles, teniendo en cuenta seis tipos diferentes (roble, duraznillo, árbol caucho, caña de azúcar, café, cascarilla y paja de arroz) y registrando la cantidad de masa en $\mathrm{kg} /$ hectárea/año utilizada para llevar a cabo procesos biomásicos. 
Tabla 4. Relación entre el poder calorífico y la energía producida de los precursores de combustibles

\begin{tabular}{|l|c|c|r|}
\hline Precursor de combustibles & $\begin{array}{c}\text { Cantidad kg/hectárea/ } \\
\text { año [ref.] }\end{array}$ & $\begin{array}{c}\text { Poder calorífico } \\
\text { MJ/kg [ref.] }\end{array}$ & $\begin{array}{c}\text { Energía producida } \\
\text { MJ/hectáreas/año }\end{array}$ \\
\hline $\begin{array}{l}\text { Roble (hojas, raíces y } \\
\text { ramas) }\end{array}$ & $1332,95[46]$ & $18,7[47]$ & 24926,2 \\
\hline $\begin{array}{l}\text { Duraznillo (hojas, raíces y } \\
\text { ramas) }\end{array}$ & $1101,41[46]$ & $18,020(\mathrm{~S})[48]$ & 19847,4 \\
\hline Árbol caucho & $1100[49]$ & $17,41[50]$ & 19151 \\
\hline Caña de azúcar (RAC) & $13000[51]$ & $14,7(\mathrm{~S})[51]$ & 191100 \\
\hline Café (Cisco) & $227[52]$ & $16,7(\mathrm{I})[53]$ & 3791 \\
\hline Cascarilla y paja de arroz & $2000[11]$ & $15,3(\mathrm{M})[53]$ & 30600 \\
\hline
\end{tabular}

Fuente: elaboración propia.

En la presente tabla 4 se evidencia que el precursor de combustible de mayor energía es la caña de azúcar, con un valor de $191100 \mathrm{MJ} /$ hectáreas anuales; la alta producción de energía se debe a la cantidad de masa utilizada que corresponde a $13000 \mathrm{~kg} / \mathrm{hectárea} / \mathrm{año}$ de residuos agrícolas de cosecha (RAC) como el bagazo, tallo verde y paja, a pesar de contar con una alta generación de energía es la que tiene el más bajo poder calorífico de $14,7 \mathrm{MJ} / \mathrm{kg}$. Por otro lado se encuentra la cascarilla y paja de arroz, con un total de $30600 \mathrm{MJ} /$ hectáreas/ año y cuenta con un poder calorífico de $15,3 \mathrm{MJ} / \mathrm{kg}$, seguido de los precursores de combustible como el roble, el cual cuenta con una energía de 24926,2 MJ/ hectáreas y un poder calorífico mayor con respeto a los demás, esto indica que al tener un alto poder calorífico la calidad de la biomasa es mejor. Luego está el duraznillo, con 19847,4 MJ/ hectáreas y su poder calorífico de 18020 $\mathrm{MJ} /$ hectáreas. La biomasa de los tipos de precursores anteriormente mencionados por medio de la combustión pueden proporcionar agua caliente y calefacción entre otras.

Por último, está el cisco del café, con una energía producida de 3791 $\mathrm{MJ} /$ hectáreas/año, esto se debe a la cantidad de masa utilidad en el informe investigado el cual utilizan un valor de $227 \mathrm{~kg} /$ hectárea/año o por su poder calorífico inferior de $16,7 \mathrm{MJ} / \mathrm{kg}$.

En la tabla 5 se observa la energía de los precursores de combustibles y la energía generada por cada una de las 
diferentes especies, también el número de cabezas de las especies que se necesitan para remplazar los precursores de combustibles investigados, en los que se escogen tres tipos de animales como cabras, vacas y cerdos.

Tabla 5. Datos para sustituir la cantidad de madera por cabezas de especies

\begin{tabular}{|c|c|c|c|c|c|c|c|}
\hline \multirow[t]{2}{*}{$\begin{array}{l}\text { Precursores de } \\
\text { combustibles }\end{array}$} & \multirow{2}{*}{$\begin{array}{c}\text { Energía de } \\
\text { los precur- } \\
\text { sores de } \\
\text { combusti- } \\
\text { bles MJ/ } \\
\text { hectáreas }\end{array}$} & \multicolumn{3}{|c|}{$\begin{array}{l}\text { Energía generada por } \\
\text { especies MJ/cabezas }\end{array}$} & \multicolumn{3}{|c|}{$\begin{array}{c}\text { Número de cabezas } \\
\text { necesarias para el remplazo } \\
\text { de los precursores cabezas/ } \\
\text { hectáreas }\end{array}$} \\
\hline & & Caprinos & Vacunos & Porcinos & Cabras & Vacas & Cerdos \\
\hline $\begin{array}{l}\text { Robles (hojas, } \\
\text { raíces y ramas) }\end{array}$ & 24926,2 & 1,7 & 5,14 & 3,02 & 14662 & 4849 & 8254 \\
\hline $\begin{array}{l}\text { Duraznillo (hojas, } \\
\text { raíces y ramas) }\end{array}$ & 19847,4 & 1,7 & 5,14 & 3,02 & 11675 & 3861 & 6572 \\
\hline Árbol caucho & 19151 & 1,7 & 5,14 & 3,02 & 11265 & 3726 & 6341 \\
\hline $\begin{array}{l}\text { Caña de azúcar } \\
\text { (RAC) }\end{array}$ & 191100 & 1,7 & 5,14 & 3,02 & 112412 & 37179 & 63278 \\
\hline Café (Cisco) & 3791 & 1,7 & 5,14 & 3,02 & 2230 & 738 & 1255 \\
\hline $\begin{array}{l}\text { Cascarilla y paja } \\
\text { de arroz }\end{array}$ & 30600 & 1,7 & 5,14 & 3,02 & 18000 & 5953 & 10132 \\
\hline
\end{tabular}

Fuente: elaboración propia.

Según los cálculos obtenidos, el precursor que genera mayor energía es la caña de azúcar con, un valor de 191100 $\mathrm{MJ} /$ hectáreas, lo que indica que se necesitan 112412 cabezas/ hectáreas de cabras para suplir la madera utilizada (ver figura1). Debemos tener en cuenta que es una cantidad muy elevada, esto se debe a la cantidad de biomasa usada según la fuente de investigación y a la generación de estiércol utilizada en el documento consultado que es de 1,6kg/cabeza; en el caso de los vacunos esta representa una menor cantidad de cabezas con un valor de 37179 vacas debido a que la generación de excremento es mayor. Por último están los porcinos, con una cifra 63278 cerdos/hectáreas. 
Por consiguiente, los precursores de café en el cual se necesitan una cantidad combustible, como lo son la cascarilla pequeña de cabezas de cabras, vacas de arroz, roble, duraznillo y árbol y cerdos con valores de 2230, 738 y caucho presentan datos elevados en 1255 respectivamente, en relación con cuanto a la cantidad de cabezas / los demás resultados, esto se debe a la hectáreas necesarias para la sustitución de la leña en comunidades rurales. Como último, se observa el cisco del la menor.

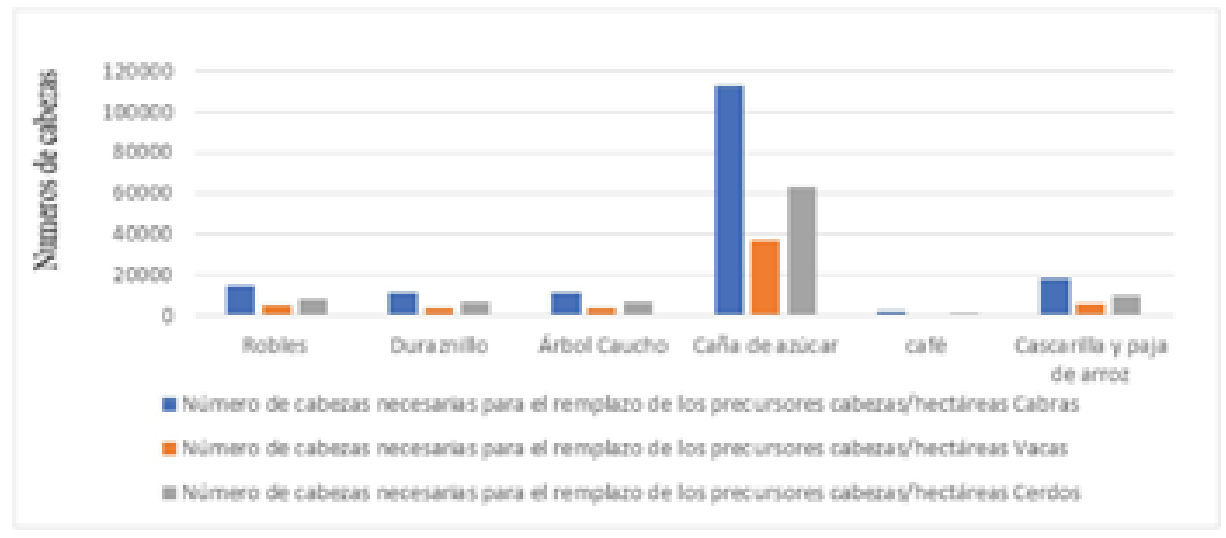

Figura 2. Cantidad de cabezas de animales (cabras, vacas y cerdos) necesarias para suplir la leña utilizadas en las comunidades rurales.

Fuente: elaboración propia.

Haciendo referencia a lo anterior, resaltamos que los $\mathrm{kg} /$ hectáreas/año tomados de los documentos consultados corresponden a áreas de bosques y de cultivos energéticos, por tal razón, al momento de comparar la cantidad de cabezas por hectáreas que se necesitan para el remplazo de los precursores, arrojan valores elevados.

\section{Conclusiones}

De acuerdo con la revisión teórica que se realizó en el presente documento, se concluye que la cantidad de excremento de las especies caprinas fueron las adecuadas debido a que cumplen con las características necesarias para la producción de biogás, puesto que, de 
acuerdo con lo consultado, se hallaron dos estudios en los que se utilizaron 40 y 80 cabezas caprinas, las cuales disponen de $27 \mathrm{~kg} /$ día y $128 \mathrm{~kg} /$ día de excremento para la producción de biogás; la comunidad Yutaho cuenta con 95 cabezas, lo que ratifica que con este número de cabezas se generaría la cantidad de estiércol necesaria para producir biogás en una comunidad.

Para la generación del biogás, el biodigestor más adecuado para implementar en comunidades rurales es el tubular, porque, de acuerdo con lo investigado, su ejecución es de bajo costo y de fácil diseño, por lo tanto, se convierte en una alternativa ideal para emplearla en el departamento de La Guajira, tomando como ventaja que el departamento cuenta con la mayor población de caprinos en Colombia, a pesar de ello no se le da un adecuado aprovechamiento.

En comunidades rurales que no tienen acceso a los servicios públicos $\mathrm{y}$, por esta razón, se ven en la necesidad de recurrir al uso de la leña para la cocción de sus alimentos, lo cual conlleva a la contaminación atmosférica y a la desforestación; por tal razón surgió la inquietud de buscar alternativas que permitan solucionar estas problemáticas, haciendo aprovechamiento de la biomasa con la que se cuenta en dichas comunidades como lo es el estiércol caprino.

\section{Referencias}

Altamirano, R. (2017). Potencial de producción energética de biogás a partir de residuos pecuarios en la región. Trujillo, Perú: La Libertad.

Arévalo, W. (2015). La biomasa: una alternativa energética proveniente de la vida misma. Nariño, Colombia: Editorial Unimar.

Badii, M., Guillén, A. y Abreu, J. (2016). Energías renovables y conservación de energía, 11(1), 141-155.

Bakirtas, T. y Gokce, A. (2018). The Relationship between Energy Consumption, Urbanization, and Economic Growth in New Emerging-Market Countries. Elsevier, 147, 110-121.

Bernal, D. y Quintero, D. (2016). Desarrollo de una propuesta para la mejora de un modelo de biodigestor anaerobio convencional a escala banco a partir de una mezcla de residuos bovinos y lodos en la finca El Recodo de Tabio, Cundinamarca. Bogotá, D. C.

Bautista, V. (2016). Evaluación de la generación de biogás a partir de excretas porcinas en la granja Agroinporc y diseño de un biodigestor. Quito.

Bolivar, J. y Hernández, Y. (2013). Análisis de viabilidad de la utilización de biomasa para la generación de energía en la sede Utopía de la Universidad de La Salle. 
Cadavid, L. y Bolaños, I. (2015). Aprovechamiento de residuos orgánicos para la producción de energía renovable en una ciudad colombiana. Energetica, 46, 23-28.

Calderón, C. (2015). Diseño de un biodigestor tubular para obtener biogás a partir de residuos orgánicos del ganado vacuno generados en la Hacienda "Santa Mónica", Guamote. Riobamba, Ecuador: Escuela Superior Politécnica de Chimborazo.

Campos, B. (2011). Methodology to determine the design and construction parameters of design of biogas installations for little farms. Revista Ciencias Técnicas Agropecuarias, 20(2), 37 -41.

Cardona, A. (2017). Agronegocios. Recuperado de https://www.agronegocios.co/ ganaderia/la-guajira-lidera-censo-en-ganado-ovino-y-caprino-2622943 (consultado el 7 de agosto del 2018).

Castaño, J. (2017). Factibilidad de la implementación de un biodigestor que transforme estiércol de porcino en biogás y biofertilizante para la venta comercial, en la finca la primavera, municipio de Lejanías, Meta.

Cendales, E. (2011). Producción de biogás mediante la codigestión anaeróbica de la mezcla de residuos cítricos y estiércol bovino para su utilización como fuente de energía renovable. Bogotá, D. C.

Chacha, J. y Flores, A. (2017). Implementación de un biodigestor piloto unifamiliar para la obtención y caracterización de biogás de uso calorífico a base de estiércol vacuno. Latacunga, Ecuador.

Chillo, J. y Paguay, S. (2015). Implementación de un biodigestor continuo para producción de biogás a partir de estiercol de ganado vacuno en la finca la poderosa. Riobamba, Ecuador.

Corporación Ambiental Empresarial: Fundación Natura (2014). Crecimiento, biomasa acumulada y carbono capturado de 25 especies de árboles y arbustos nativos de la cordillera oriental colombiana. Bogotá, D.C.

Cruz, A. (2014). Desarrollo de un biodigestor casero como alternativa para la generación de biogás empleando estiércol bovino. Terreon-Coahuila, México.

Cunalata, J. (2017). Diseño de un sistema de transporte de biogás para uso doméstico en la hacienda San Francisco, Latacunga.

Durazno, A. (2018). Valoración de estiércol bovino y porcino en la producción de biogas en un biodigestor de producción por etapas. Cuenca, Ecuador.

EPM (2018). Parque Eólico Jepírachi. [Página web]. Recuperado de https://www. epm.com.co/site/home/institucional/nuestras-plantas/energia/parque-eolico (consultado el 8 de agosto del 2018).

Fernández, J., Gutiérrez, F., Del Río, P., San Miguel, G., Bahillo, A., Sánchez, J., Ballesteros, M., Vásquez, J., Rodríguez, L. y Aracil, J. (2015). Tecnologías para el uso y transformación de biomasa energética. Madrid: Mundi-Prensa.

Follari, J. y Torres, M. (2014). Un biodigestor de boñigas de cabra calefaccionado con colectores planos.

Garfí, M., Martí, J., Garwood, A. y Ferrer, I. (2016). Household anaerobic digesters for biogas production in Latin America:A review. Elserver, 60, 599-614. 
Inca, J (2016). Diseño de un biodigestor para la obtención de biogás a partir de las excretas de las gallinas provenientes de la granja avícola "Bilbao" en la parroquia Cotaló-Pelileo, Riobamba, Ecuador.

Lara, M. (2016). Diseño de un biodigestor para la producción de biogás generado por las excretas de ganado vacuno, en el criadero “Jersey Chugllin". Riobamba, Ecuador:

Miller, J. y Visicdi, L. (2016). Innovación en energía limpia en América Latina. Socioteca.

Ministerio de Agricultura, Ganadería y pesca. Presidencia de la Nación (2013). La caña de azúcar como cultivo energético. Tucumán, Argentina.

Navarro, L. (2015). Diseño y construcción de un biodigestor anaeróbico para el aprovechamiento energético de la biomasa y uso experimental para la Universidad Francisco de Paula Santander Ocaña, Santander.

Ordóñez, K. (2010). Producción de biogás a base de desechos gropecuarios (estiércol de cabra y pasto). Torreón.

Oviedo, J., Badii, M., Guillen, A. y Lugo, O. (2015). Historia y uso de energías renovables. Daena: International Journal of Good Conscience, 10(1), 1-18.

Pérez, D. (2017). Omicrono, la tecnología de El Español. Recuperado de https://omicrono.elespanol.com/2017/02/paises-masinversoresenergiasrenovables/. (consultado el 7 de agosto del 2018).

Pérez, L. y Salazar, S. (2015). Elaboración de una guía ambiental para el manejo limpio en el aprovechamiento energético de la biomasa residual. Bogotá D.C.
Quintero, J. y Quintero, L. (2015). Perspectivas del potencial energético de la biomasa en el marco goblal y latinoamericano. Gestión y Ambiente.

Quintero, A., Walkiria, C., Fernández, L. y De Calzadilla, J. (2010). Diagnóstico del sistema de producción-comercialización del ganado caprino-ovino en el departamento de La Guajira, Colombia. Aplicación del Escalamiento Óptimo. Revista Ciencias Técnicas Agropecuarias, 19(2), 57-64.

Rodríguez, D. y García, A. (2017). Diseño y construcción de un biodigestor para la producción de biogás a partir de heces caninas. Bogotá, D.C.

Rodríguez, N. y Zambrano, D. (2010). Los subproductos del café: fuente de energía renovable, Caldas.

Shahsavari, A. y Akbari, M. (2018). Potential of solar energy in developing countries for reducing energy-related emissions. Renewable and Sustainable Energy Reviews, 90, 275-291.

Shahbaz, M., Zakaria, M., Hussain, S. Shahzad, J. y Mahalik, M. (2018). The energy consumption and economic growth nexus in top ten energy-consuming countries: Fresh evidence from using the quantile-on-quantile approach. Energy economics, 71, 282-301, 2018.

Smitha, M., Schroenn, J. y Blignaut, J. (2014). The financial and economic feasibility of rural household biodigesters for poor communities in South Africa. Elsevier, 34(2), 352-362.

Solano, J. (2015). Energía en América Latina: una aproximación prospectiva. Pizarrón 
Latinoamericano: Realidad y Contexto de América Latina, 3(3), pp. 23-33, 2015.

Toscano, T. C. (2016). Diseño de un biodigestor anaeróbico para la obtención de biogás, a partir de las excretas de ganado vacuno en el Rancho Guadalupe, en el Cantón Mocha, Provincia de Tungurahua en el 2015. Riobamba-Ecuador: Escuela Superior Politécnica de Chimborazo.

Vergara, W., Isbell, P., Ríos, A. Gómez, J. y Alves, L. (2014). Beneficios para la sociedad de la adopción de fuentes renovables de energía en América Latina y el Caribe.

Villanueva, M. (2012). Los determinantes del consumo energético en España: ¿se ha mejorado la eficiencia energética? Papeles de Economía Española, 134, 196-210.
Yauyo, L. (2016). Elaboración de un biodigestor piloto tubular para el manejo de estiércol porcino, en una de las viviendas de la asociación agropecuaria los lúcumos de Pachacamac, Villa El Salvador.

Zareei, S. (2018). Evaluation of biogas potential from livestock manures and rural wastes using GIS in Iran. Elsevier, 118, 351-356.

Zhang, T., Liu, L., Song, Z., Ren, G., Feng, Y., Han, X. y Yang, G. (2013). Biogas Production by Co-Digestion of Goat Manure with Three Crop Residues. Plos-ONE, 8(6). 